\section{A) Check for updates}

Cite this: Food Funct., 2018, 9, 6287

\title{
Maltol, a food flavor enhancer, attenuates diabetic peripheral neuropathy in streptozotocin-induced diabetic rats
}

\author{
Nan Guo, ${ }^{a, b}$ Caina Li, ${ }^{a, b}$ Quan Liu, ${ }^{a, b}$ Shuainan Liu, ${ }^{a, b}$ Yi Huan, ${ }^{a, b}$ Xing Wang, ${ }^{a, b}$ \\ Guoliang Bai, ${ }^{a, b}$ Miaomiao Yang, ${ }^{a, b}$ Sujuan Sun, ${ }^{a, b}$ Caimin $X u^{c}$ and \\ Zhufang Shen iD *a,b
}

Scope: Maltol (3-hydroxy-2-methy-4-pyrone), a potent antioxidative agent, typically is used to enhance flavor and preserve food. This study evaluated its effects on preventing diabetic peripheral neuropathy (DPN) in streptozotocin (STZ)-induced diabetic rats and explored its mechanisms. Methods and results: We intraperitoneally injected Sprague-Dawley (SD) rats with STZ (65 $\mathrm{mg} \mathrm{kg}^{-1}$, ip) and treated the rats with different doses of maltol after 4 weeks of injection. During treatment, we evaluated motor nerve conduction velocity (MNCV) and thermal and mechanical hyperalgesia and assayed the oxidative stress, $\mathrm{Na}^{+}-\mathrm{K}^{+}-$ ATPase activity, and apoptosis. Repeated treatment with maltol for 12 weeks significantly improved thermal and mechanical hyperalgesia, increased the MNCV, elevated the $\mathrm{Na}^{+}-\mathrm{K}^{+}$-ATPase activity, and ameliorated oxidative stress and apoptosis in STZ-induced diabetic rats. We coincubated RSC96 cells, a Schwann cell line, with maltol and hydrogen peroxide $\left(\mathrm{H}_{2} \mathrm{O}_{2}, 0.6 \mathrm{mM}\right)$. Evidently, maltol increased cell viability and inhibited apoptosis after injury by $\mathrm{H}_{2} \mathrm{O}_{2}$. Conclusions: Maltol was demonstrated to prevent DPN development and may provide a new alternative for the treatment of DPN.

Received 9th October 2018 Accepted 16th October 2018 DOI: $10.1039 /$ c8fo01964a rsc.li/food-function mechanisms. ${ }^{1}$ Despite therapeutic advances, no drugs have targeted any of these four mechanisms simultaneously. Antioxidants may be useful, such as $\alpha$-lipoic acid (Nevralip Retard $\circledast$, Medical Pharmaquality, Athens, Greece), a potent antioxidant. Through animal experiments and clinical trials, $\alpha$-lipoic acid has been demonstrated to be effective in treating $\mathrm{DPN}^{2}$ and many other antioxidants, such as taurine, acetyl-carnitine, M40403, and $\beta$-carotene, also have been demonstrated to be effective in ameliorating the development of DPN. ${ }^{3}$

Maltol (3-hydroxy-2-methy-4-pyrone), a type of aromatic compound, exists in high concentrations in red ginseng, which is manufactured by steaming and drying fresh ginseng, a traditional herbal medicine. ${ }^{4}$ Currently, maltol is used extensively as a safe flavoring agent and food preservative, and it is always found in heat-processed food. ${ }^{5}$ As a well-known metal ion chelator, maltol is widely used in the field of catalysis, cosmetics, and medicine. Furthermore, studies have demonstrated that maltol was a strong antioxidant ${ }^{6}$ and it significantly inhibited the production of AGEs. ${ }^{7}$ Maltol also could defend against oxidation-induced damage to nerve cells, kidneys, and the liver by scavenging free radicals in the body, ${ }^{8,9}$ and effectively inhibiting the apoptosis induced by hydrogen peroxide $\left(\mathrm{H}_{2} \mathrm{O}_{2}\right),{ }^{10}$ which has attracted increasing attention in the research and development of drugs. Moreover, there are still no reports on the use of maltol to treat DPN. 
Therefore, the current study prospectively investigated the effects of maltol on DPN following treatment for 12 weeks in streptozotocin (STZ)-induced diabetic rats and preliminarily explored the mechanisms in vivo and in vitro, to supply the experimental basis to extend its application.

\section{Materials and methods}

\subsection{Animals}

Male Sprague-Dawley (SD) rats (150-170 g; Beijing HFK Bioscience Co. Ltd, Beijing, China) were kept in a temperatureand humidity-controlled environment with a $12 \mathrm{~h}$ light/dark cycle and fed food and water ad libitum. The Experimental Animal Welfare Ethics Committee of the Institute of Materia Medica (Chinese Academy of Medical Sciences and Peking Union Medical College) under No. 00001042 approved all of the protocols for this research, and all of the rats were handled according to the commonly accepted " $3 \mathrm{R}$ " and standards and guidelines for laboratory animals (GB14925-2001 and MOST 2006a) established by the People's Republic of China.

\subsection{Cells}

RSC96 cells, the rat Schwann cell line, were purchased from the Cell Resource Center of the Shanghai Institutes for Biological Sciences, Chinese Academy of Sciences (Shanghai, China) and cultured in Dulbecco's modified Eagle's Medium (DMEM; Gibco Laboratories, Gaithersburg, MD, USA) plus $100 \mathrm{U} \mathrm{mL}^{-1}$ penicillin (Sigma-Aldrich, St Louis, MO, USA), $100 \mathrm{mg} \mathrm{mL}{ }^{-1}$ streptomycin (Sigma-Aldrich) and 10\% (v/v) fetal bovine serum (FBS; Gibco) at $37{ }^{\circ} \mathrm{C}$ under a $5 \%$ carbon dioxide-humidified atmosphere.

\subsection{Experimental design and treatment}

In vivo, after 3 days of acclimatisation, we intraperitoneally injected all of the rats with STZ $\left(65 \mathrm{mg} \mathrm{kg}{ }^{-1}\right.$, ip; SigmaAldrich) dissolved in citrate buffer $\left(0.1 \mathrm{~mol} \mathrm{~L}^{-1}, \mathrm{pH} 4.5\right)$ to induce hyperglycemia. We determined the fasting blood glucose (FBG) levels to verify the occurrence of diabetes 3 days later. Four weeks after the verification of diabetes, we determined the thermal and mechanical pain thresholds and the erythrocytic $\mathrm{Na}^{+}-\mathrm{K}^{+}$-ATPase activity of rats with blood glucose $\geq 11.1 \mathrm{mmol} \mathrm{L}^{-1}$, and then we divided the rats into five groups $(n=12)$ : the vehicle-treated group (Con), the maltol-treated groups $\left(50,100\right.$, and $\left.200 \mathrm{mg} \mathrm{kg}^{-1}\right)$, and the $\alpha$-lipoic acid-

Table 1 Data for the grouping of STZ-induced diabetic rats

\begin{tabular}{lllll}
\hline Group & $\begin{array}{l}\text { Dose } \\
\left(\mathrm{mg} \mathrm{kg}^{-1}\right)\end{array}$ & $\begin{array}{l}\text { Withdrawal } \\
\text { latency }(\mathrm{s})\end{array}$ & $\begin{array}{l}\text { Withdrawal } \\
\text { threshold }(\mathrm{g})\end{array}$ & $\begin{array}{l}\mathrm{Na}^{+}-\mathrm{K}^{+}-\mathrm{ATPase} \\
\left(\mathrm{U} \mathrm{mL}^{-1}\right)\end{array}$ \\
\hline Nor & - & $15.8 \pm 0.8^{* *}$ & $43.0 \pm 0.7^{* *}$ & $7.4 \pm 0.3^{* *}$ \\
Con & - & $10.0 \pm 0.5$ & $27.4 \pm 1.1$ & $5.1 \pm 0.5$ \\
ALA & 100 & $10.0 \pm 0.5$ & $27.4 \pm 1.1$ & $5.0 \pm 0.7$ \\
Maltol & 50 & $9.8 \pm 0.5$ & $27.5 \pm 1.2$ & $5.0 \pm 0.4$ \\
& 100 & $10.0 \pm 0.5$ & $27.4 \pm 1.2$ & $4.9 \pm 0.5$ \\
& 200 & $10.0 \pm 0.5$ & $27.4 \pm 1.2$ & $4.8 \pm 0.5$
\end{tabular}

Data expressed as mean $\pm \mathrm{SEM}, n=10-12,{ }^{* *} p<0.01 v s$. Con. treated group (100 mg kg ${ }^{-1}$; see Table 1$)$. We adopted normal age-matched SD rats as the healthy control (Nor, $n=10$ ) and $\alpha$-lipoic acid served as the positive reference. We treated the rats with maltol or $\alpha$-lipoic acid dissolved in $0.5 \%$ carboxymethyl cellulose and sodium (CMC-Na) solution by gavage once daily for 12 weeks after division, and the Nor and Con rats were orally administered $0.5 \% \mathrm{CMC}-\mathrm{Na}$ solution. Insulin treatment was not given. We dynamically monitored the fasting blood glucose (FBG) levels and body weight (BW) during the treatment and evaluated other parameters, including motor nerve conduction velocity (MNCV), thermal and mechanical hyperalgesia, $\mathrm{Na}^{+}-\mathrm{K}^{+}$-ATPase activity, total antioxidant capacity (TAOC), malondialdehyde (MDA), glutathione (GSH), and superoxide dismutase (SOD) at the end of the experiment (Fig. 1A).

In vitro, we incubated RSC96 cells with $\mathrm{H}_{2} \mathrm{O}_{2}(0.6 \mathrm{mM})$ to induce oxidative damage. We separately coincubated maltol at concentrations 0.1 and $0.5 \mathrm{mM}$, as well as $\alpha$-lipoic acid at a
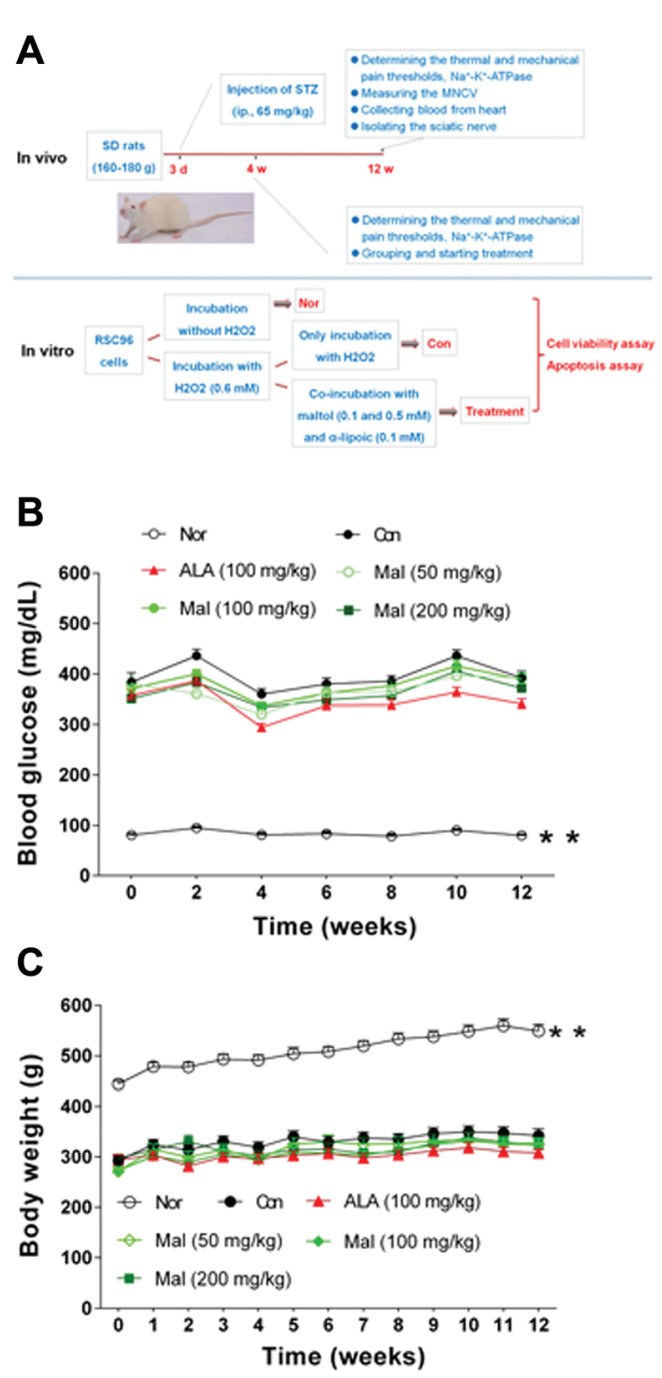

Fig. 1 Experimental scheme and the effects of treatment with maltol on fasting blood glucose and body weight in STZ-induced diabetic rats. (A) Experimental scheme, (B) blood glucose, and (C) body weight. Data expressed as mean $\pm \mathrm{SEM}, n=11-12,{ }^{*} P<0.01$ vs. Con. 
concentration of $0.1 \mathrm{mM}$ with $\mathrm{H}_{2} \mathrm{O}_{2}$ in RSC96 cells. We used cells without any treatment as the normal control (Nor). Then, we assayed cell viability by CCK8 and evaluated apoptosisrelated proteins by western blot and real-time polymerase chain reaction (PCR; Fig. 1A).

\subsection{Measurement of FBG and BW}

To measure FBG, all of the rats were fasted for $4 \mathrm{~h}$ with water ad libitum, and then we determined the blood glucose levels using the glucose-oxidase method every 2 weeks. The BW was documented once weekly during treatment without a withdrawal of diet.

\subsection{Measurement of $\mathrm{MNCV}$}

After treatment for 12 weeks, all of the rats were anesthetized with sodium pentobarbital (60 $\mathrm{mg} \mathrm{kg}^{-1}$, ip). We stripped the left sciatic nerve to receive a stimulation at the proximal end by square-wave pulses (duration: $0.01 \mathrm{~ms}$, intensity: $1 \mathrm{~V}$ ) delivered through bipolar recording electrodes, and the action potential at the distal end was recorded using the BL-420S biomechanical system (Chengdu Taimeng Technology Co., Ltd, Chengdu, China). We calculated $\operatorname{MNCV~}\left(\mathrm{m} \mathrm{s}^{-1}\right)$ as follows: (the distance between the stimulating and recording electrode)/ latency. ${ }^{11}$

\subsection{Measurement of the thermal and mechanical hyperalgesia}

After treatment for 12 weeks, we monitored the thermal and mechanical hyperalgesia by a plantar test and recorded the latency or threshold of paws withdrawal after stimulation using Hargreaves Apparatus 37370 and Dynamic Plantar Aesthesiometer 37450 (Ugo Basile S.R.L., Comerio, Italy). In short, we placed the rats separately in six cages $(17 \times 11.5 \times 14 \mathrm{~cm})$ and stimulated the left hind paws with infrared light (cutoff period set at $25 \mathrm{~s}$ ) or a mechanical needle (biting strength set at $50 \mathrm{~g}$ ). The equipment automatically recorded paw withdrawal latency as soon as the paws withdrew. ${ }^{12}$ We assessed each rat five times with a 5 min interval between trials and took the mean value as withdrawal threshold or latency.

\subsection{Measurement of $\mathrm{Na}^{+}-\mathrm{K}^{+}$-ATPase activity}

After treatment for 12 weeks, all of the rats were killed by drawing blood from the heart and the sciatic nerves were isolated. Then, we determined the $\mathrm{Na}^{+}-\mathrm{K}^{+}$-ATPase activities in erythrocytes and the sciatic nerves as follows. For the determination in erythrocytes, we collected $10 \mu \mathrm{l}$ of whole blood and added it to $240 \mu \mathrm{l}$ of distilled water to mix and immediately determine the $\mathrm{Na}^{+}-\mathrm{K}^{+}$-ATPase activity. For the determination in sciatic nerves, we first cut one side of the sciatic nerves into pieces with ophthalmic scissors in cold saline (100 $\mathrm{mg}: 1800 \mu \mathrm{l}$ ) and homogenized the nerves with Beed Ruptor 24 Elite (OMNI International, Kennesaw, GA, USA). Then, we centrifuged the homogenate at $4{ }^{\circ} \mathrm{C}, 3500 \mathrm{rpm}$ for $15 \mathrm{~min}$ and collected the supernatant to be used. The determination and calculation of $\mathrm{Na}^{+}-\mathrm{K}^{+}$-ATPase activities were pro- cessed using commercial kits bought from Nanjing Jiancheng Bioengineering Institute (Nanjing, China).

\subsection{Measurement of TAOC, MDA, GSH and SOD}

At the end of the experiment, we isolated the serum by centrifugation at $4{ }^{\circ} \mathrm{C}, 3000 \mathrm{rpm}$ for $10 \mathrm{~min}$. We then determined the content of TAOC, MDA, GSH, and SOD in serum and sciatic nerve homogenates with commercial kits bought from Nanjing Jiancheng Bioengineering Institute (Nanjing, China) according to the manufacturer's instructions.

\subsection{Measurement of caspase-3 activity}

After treatment for 12 weeks, we also collected the dorsal root ganglia (DRG) under a stereoscope to measure the caspase-3 activities with a caspase-3 colorimetric assay kit (Applygen Technologies Inc., Beijing, China). First, we cut the DRG into pieces with ophthalmic scissors in a lysis buffer (3-10 mg: $100 \mu \mathrm{l}$ ) and homogenized with Beed Ruptor 24 Elite (OMNI International). Then, we collected the supernatant by centrifugation at $4{ }^{\circ} \mathrm{C}, 12000 \mathrm{~g}$ for $10 \mathrm{~min}$. We quantified the protein in the supernatant following the Bradford method (Applygen Technologies Inc.). We calculated the caspase-3 activities with protein correction.

\subsection{CCK-8 assay}

We planted RSC96 cells into a 96-well plate (15 000 cells per well) and cultured the cells overnight in DMEM plus $100 \mathrm{U} \mathrm{mL}^{-1}$ penicillin, $100 \mathrm{mg} \mathrm{mL}^{-1}$ streptomycin and $10 \%$ (v/v) FBS. Six hours after treatment, as described in Section 2.3, we added $10 \mu \mathrm{l}$ of the CCK-8 assay reagent (Dojindo Molecular Technologies, Inc., Kumamoto, Japan) and incubated the cells for another $3 \mathrm{~h}$. Then, we measured the absorbance at $450 \mathrm{~nm}$ and used the optical density (OD) values on the $y$-axis to express the cell viability.

\subsection{Western blot}

We evaluated the apoptosis-related proteins, including Bcl-XL, BAX, and caspase-3 in sciatic nerves of STZ-induced diabetic rats after treatment for 12 weeks, and evaluated Bcl-XL in RSC96 cells cultured and treated as noted in Section 2.10, with western blot. First, we extracted total protein from one side of the sciatic nerve and RSC96 cells planted in 6-well plates (200 000 cells per well) with RIPA lysate plus inhibitors of protease and protein phosphatase (Applygen Technologies Inc.), followed by protein quantification using a BCA assay kit (Applygen Technologies Inc.). We electrophoretically separated equal amounts of protein by sodium dodecyl sulfate polyacrylamide gel electrophoresis (SDS-PAGE) and transferred the protein to a polyvinylidene difluoride (PVDF) membrane (Millipore, Darmstadt, Germany). After blocking for $1 \mathrm{~h}$ with $5 \%$ nonfat milk, we separately incubated the PVDF membrane with primary antibodies, including Bcl-XL, BAX, caspase-3, and $\beta$-actin $(1: 1000$, Cell Signaling Technology, Danvers, MA, USA) at $4{ }^{\circ} \mathrm{C}$ overnight, followed by incubation with mouse anti-rabbit secondary antibody conjugated with horseradish peroxide (1:1000, Applygen Technologies Inc.) after washing. 
We visualized the protein bands through an enhanced chemiluminescence detection system (ChemiScope 2850; Clinx Science Instruments, Shanghai, China) and quantified the relative band densities using $\beta$-actin as the internal standard.

\subsection{Quantitative real-time PCR}

We also evaluated the apoptosis-related genes, including BAG4, BAX, and caspase-3 in the sciatic nerve and evaluated BAX and caspase-3 in RSC96 cells with real-time PCR. First, we extracted total RNA from one side of the sciatic nerve and RSC96 cells planted and treated as noted in Section 2.11 using TRIzol reagent (Invitrogen, Carlsbad, CA, USA). Then, we synthesized the cDNA using a VigoScript first strand cDNA synthesis kit (Vigorous Biotechnology Beijing Co., Ltd, Beijing, China). We conducted quantitative real-time PCR with SYBR Green Master Mix (Takara, Dalian, China) using the 7900HT fast real-time PCR system (Applied Biosystems, Foster City, CA, USA) according to the following protocol: 1 cycle at $95{ }^{\circ} \mathrm{C}$ for $30 \mathrm{~s}$, then 40 cycles at $95{ }^{\circ} \mathrm{C}$ for $5 \mathrm{~s}$, and $60{ }^{\circ} \mathrm{C}$ for 31 s. Glyceraldehyde 3-phosphate dehydrogenase (GAPDH) served as the internal standard. We used the following primer sequences: BAG4, forward 5'-GCGTTTCCTCACTCGTGACA-3' and reverse 5'-AAGGGGGTTAGGACGGC TAC-3', BAX forward 5'-TT GCTACAGGGTTTCATCCAGG- ${ }^{\prime}$ ' and reverse 5'-CACTCGCT CAGCTTCTTGGT-3', caspase-3 forward 5'-GGAGCTTGGA ACGCGAAGAA- $3^{\prime}$ and reverse 5'-ACACAAGCCCATTTCAGGGT- 3 ', and GAPDH forward $5^{\prime}$-CCTGTTGCTGTAGCCGTATTCA- 3 ' and reverse 5'-CCAGGTT GTCTCCTGCGACTT-3'.

\subsection{Statistical analysis}

All of the results were presented as mean \pm SEM. We analyzed data through one-way analysis of variance with Bonferroni's correction and Student's $t$-test. We defined statistical significance as $P<0.05$.

\section{Results and discussion}

\subsection{Effects of maltol on FBG and BW in STZ-induced diabetic rats}

In comparison with nondiabetic rats (Nor), STZ-induced diabetic rats displayed about 4.5 -fold increase in FBG levels and 1.6-fold decrease in BW during the treatment, suggesting the occurrence of diabetes. In comparison with STZ-induced diabetic rats (Con), repeated treatment with maltol ranging from $50 \mathrm{mg} \mathrm{kg}^{-1}$ to $200 \mathrm{mg} \mathrm{kg}{ }^{-1}$ for 12 weeks produced some changes in the FBG level but with fluctuations (Fig. 1B). Repeated treatment with maltol had no effect on BW during the experiment (Fig. 1C). These results suggested that maltol had no significant influences on controlling blood glucose levels and body weight.

STZ-induced diabetic rats are a commonly used model to explore the mechanisms of neuropathy and to evaluate the effects of potential therapeutics. One study reported that DPN developed within 6 weeks after the induction of diabetes. ${ }^{13}$ Researchers have opted to start drug intervention at 4 or
8 weeks after the induction of diabetes. ${ }^{3,14}$ We divided STZinduced diabetic rats according to the thermal and mechanical pain thresholds and the erythrocytic $\mathrm{Na}^{+}-\mathrm{K}^{+}$-ATPase activity 4 weeks after the induction of diabetes, when all three markers were significantly decreased in comparison with nondiabetic rats (Table 1), suggesting pain hyperalgesia, accumulation of intra-axonal $\mathrm{Na}^{+}$, and nodal axonal swelling. ${ }^{15}$

Uncontrolled chronic hyperglycemia in STZ-induced diabetic rats was the source of DPN, and keeping the blood glucose level in a suitable range without a large fluctuation significantly decreased the rate of DPN. Thus, in the present study, we first investigated the influences of maltol on FBG and BW. We found that maltol $\left(200 \mathrm{mg} \mathrm{kg}^{-1}\right)$ produced some changes in the FBG level but with fluctuations during the treatment, which we thought may have resulted from the states of the animals before the measurement was taken, such as food intake, water consumption, and $\beta$-cell function. Repeated treatment with maltol did not influence the BW of STZ-induced diabetic rats.

\subsection{Effects of maltol on MNCV in STZ-induced diabetic rats}

In comparison with nondiabetic rats (Nor), STZ-induced diabetic rats (Con) showed decreased MNCV, suggesting the occurrence of peripheral neuropathy. In comparison with STZinduced diabetic rats (Con), repeated administration of maltol at doses of 100 and $200 \mathrm{mg} \mathrm{kg}^{-1}$ for 12 weeks improved the MNCV in a dose-dependent manner by $34.0 \%(p<0.05)$ and $58.2 \%(p<0.01)$, respectively, and the MNCV of rats in the maltol-treated group at a dose of $200 \mathrm{mg} \mathrm{kg}^{-1}$ showed no difference from that of nondiabetic rats (Nor), suggesting the improvement in peripheral nerve function (Fig. 2).

The slowing of a large fiber nerve conduction velocity (NCV) has been a gold standard for defining neuropathy in patients with type 2 diabetes mellitus and for assessing the efficacy of potential therapeutics. The slowing of MNCV and sensory NCV (SNCV) also was an early feature of diabetic mice and may be detected within weeks of the onset of diabetes. ${ }^{16}$ In our study, the MNCV of STZ-induced diabetic rats decreased by $47.9 \%$ compared with nondiabetic rats after 16 weeks of untreated diabetes. The increase in MNCV following maltol treatment at a dose of $200 \mathrm{mg} \mathrm{kg}^{-1}$ to a level that was not statistically

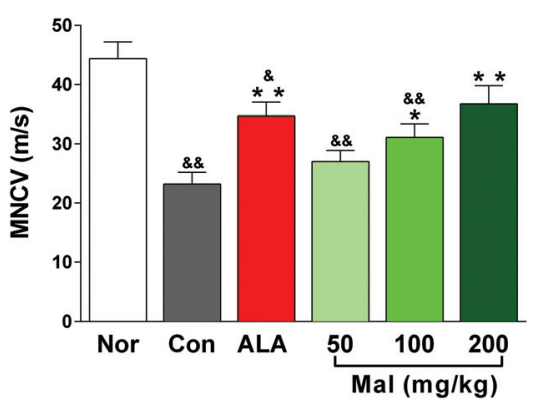

Fig. 2 Effect of treatment with maltol on the MNCV in STZ-induced diabetic rats. Data expressed as mean \pm SEM, $n=10-12,{ }^{\&} P<0.05$, \&\& $P<0.01$ vs. Nor; ${ }^{\star} P<0.05$, ** $P<0.01$ vs. Con. 
different from that of nondiabetic rats indicated its benefits in treating DPN. In addition, as a potent antioxidant, $\alpha$-lipoic acid has been a useful clinical option for patients with DPN, which also significantly reduced the oxidative-stress-induced injury and improved the NCV and blood flow. ${ }^{2,17}$

\subsection{Effects of maltol on thermal and mechanical hyperalgesia in STZ-induced diabetic rats}

In comparison with nondiabetic rats (Nor), STZ-induced diabetic rats exhibited decreased withdrawal threshold and latency in response to mechanical and thermal stimulations, indicating evident pain hyperalgesia. In comparison with STZinduced diabetic rats (Con), repeated administration of maltol at doses of 50,100, and $200 \mathrm{mg} \mathrm{kg}^{-1}$ for 12 weeks significantly extended the withdrawal threshold and latency following mechanical stimulation by $18.1 \%, 24.0 \%$, and $27.7 \%$, and thermal stimulation by $47.2 \%, 87.9 \%$, and $100.0 \%$, respectively. Furthermore, the withdrawal thresholds and latency of rats in the groups of maltol at doses of 100 and $200 \mathrm{mg} \mathrm{kg}^{-1}$ was equivalent to that of nondiabetic rats (Nor), suggesting amelioration of the pain hyperalgesia (Fig. 3).

DPN, a major cause of foot ulceration and limb loss for patients with diabetes, has been associated with sensory loss or abnormal sensations, such as hyperalgesia, allodynia and spontaneous pain. Thermal and mechanical hyperalgesia have been a clinical phenomenon in early DPN, whereas hypoalgesia has been found in advanced $\mathrm{DPN} ;{ }^{18}$ however, the mechanisms of abnormal nociception and the time for turnover of hyperalgesia to hypoalgesia in diabetes have not been well understood. In our study, we found that the thermal and mechanical pain thresholds of STZ-induced diabetic rats significantly decreased by $51.2 \%$ and $24.9 \%$ after 16 weeks of untreated diabetes compared with nondiabetic rats, suggesting hyperalgesia. These results were consistent with previous studies, wherein hyperalgesia has been reported after 6,8 , and 12 weeks of hyperglycemia. ${ }^{19-21}$ Many studies, however, have still reported hypoalgesia. For example, Davidson et al. found that STZ-induced diabetic rats displayed thermal hypoalgesia after 16 weeks of untreated diabetes, and the high-fat-fed/low- dose STZ-induced diabetic rats showed thermal hypoalgesia after 8 weeks of a high fat diet and 16 weeks of hyperglycemia induced by STZ, ${ }^{2,23}$ whereas Stavniichuk et al. reported that STZ-induced diabetic C57Bl6/J mice exhibited thermal hypoalgesia but tactile allodynia. ${ }^{14}$

Behavioral responses to mechanical touch may incorporate the function of large myelinated sensory fibers that terminated in the dermis, whereas the responses to thermal stimulation were transduced by small sensory C fibers that terminated in the epidermis. Calcutt et al. reported that diabetic rodents developed increased sensitivity to a series of manual von Frey filaments within 2-4 weeks of hyperglycemia and an initial paw thermal hyperalgesia that progressed to hypoalgesia without insulin therapy. ${ }^{24,25}$ Kambiz et al. found that STZinduced WAG/RijHsd diabetic rats did not display significantly different responses to mechanical stimulation after 4 weeks of diabetes. These rats, however, developed evident hyposensitivity after 6 and 8 weeks of diabetes and showed remarkable heat hypersensitivity in the hot plate test at 4,6 , and 8 weeks after the induction of diabetes. ${ }^{26}$ Thus, we deemed the behavioral responses to thermal and mechanical stimulation of rodents to be complicated, which may have varied as a result of the duration of diabetes, blood glucose level, and animal species etc. among different labs.

\subsection{Effects of maltol on $\mathrm{Na}^{+}-\mathrm{K}^{+}$-ATPase activities in STZ-induced diabetic rats}

We considered the $\mathrm{Na}^{+}-\mathrm{K}^{+}$-ATPase activity to be an index of microvascular blood supply and peripheral nerve injury. In comparison with nondiabetic rats (Nor), STZ-induced diabetic rats (Con) displayed decreased $\mathrm{Na}^{+}-\mathrm{K}^{+}$-ATPase activities in erythrocytes and sciatic nerves. In comparison with STZinduced diabetic rats (Con), repeated treatment with maltol at doses of 50,100 , and $200 \mathrm{mg} \mathrm{kg}^{-1}$ for 12 weeks increased the erythrocytic $\mathrm{Na}^{+}-\mathrm{K}^{+}$-ATPase activity in a dose-dependent manner by $19.2 \%, 24.8 \%$, and $28.6 \%$, respectively ( $p<0.01)$. Meanwhile, maltol at doses of 100 and $200 \mathrm{mg} \mathrm{kg}^{-1}$ also evidently increased the $\mathrm{Na}^{+}-\mathrm{K}^{+}$-ATPase activity in sciatic nerves by $13.9 \%(p<0.05)$ and $16.8 \%(p<0.01)$, respectively.
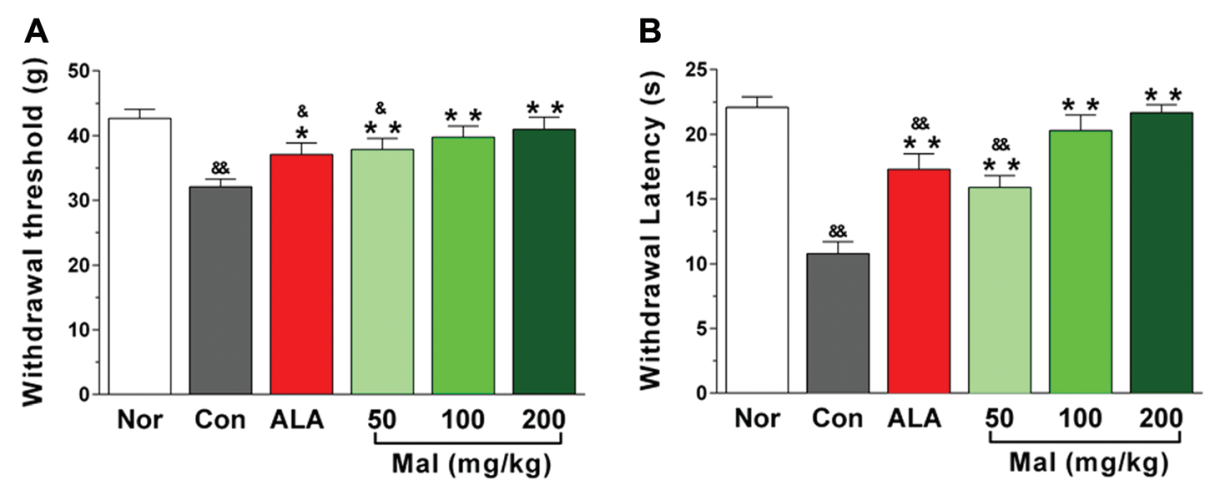

Fig. 3 Effects of treatment with maltol on the sensitivity of plantar to mechanical and thermal stimulations in STZ-induced diabetic rats. (A) Plantar withdrawal threshold to mechanical stimulations and (B) plantar withdrawal latency to thermal stimulations. Data expressed as mean \pm SEM, $n=$ $11-12{ }^{\&} P<0.05$, ${ }^{\text {\& }} P<0.01$ vs. Nor; ${ }^{*} P<0.05$, ${ }^{* *} P<0.01$ vs. Con. 
A

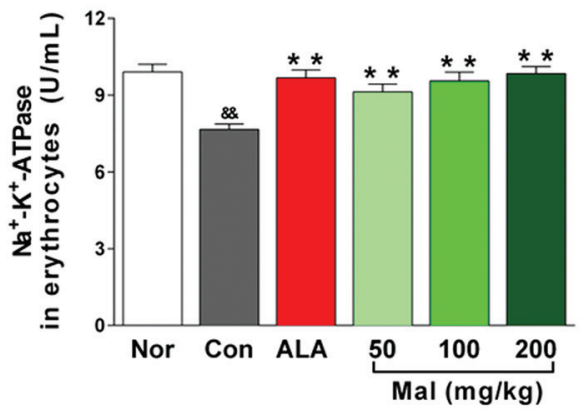

B

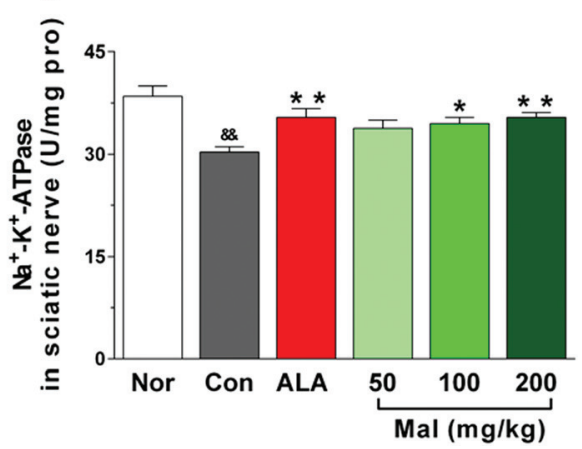

Fig. 4 Effects of treatment with maltol on the $\mathrm{Na}^{+}-\mathrm{K}^{+}$-ATPase activities in erythrocytes and the sciatic nerve of STZ-induced diabetic rats. (A) $\mathrm{Na}^{+}-\mathrm{K}^{+}$-ATPase activity in erythrocytes and (B) $\mathrm{Na}^{+}-\mathrm{K}^{+}$-ATPase activity in the sciatic nerve. Data expressed as mean $\pm \mathrm{SEM}, n=6$, \&\& $P<0.01$ vs. Nor; ${ }^{*} P<0.05,{ }^{* *} P<0.01$ vs. Con.

Furthermore, the $\mathrm{Na}^{+}-\mathrm{K}^{+}$-ATPase activity in both erythrocytes and sciatic nerves after treatment with maltol were not different from that of nondiabetic rats (Nor) (Fig. 4).

$\mathrm{Na}^{+}-\mathrm{K}^{+}$-ATPase is a ubiquitous membrane-associated enzyme that is expressed in most erythrocytes. Early studies have shown that diabetes induced a parallel decrease of $\mathrm{Na}^{+}-$ $\mathrm{K}^{+}$-ATPase activity in erythrocytes and sciatic nerve, and monitoring the $\mathrm{Na}^{+}-\mathrm{K}^{+}$-ATPase activity in erythrocytes seemed to reflect the activity in the peripheral nerve. ${ }^{27}$ Furthermore, decreased $\mathrm{Na}^{+}-\mathrm{K}^{+}$-ATPase activity in erythrocytes may be responsible for the decreased cell deformability and NCV and impaired nerve histology. ${ }^{28}$ Therefore, the erythrocytic $\mathrm{Na}^{+}-\mathrm{K}^{+}$ATPase activity could be a potential marker for a predisposition to diabetic neuropathy. ${ }^{29}$ Our study found that long-term treatment with maltol significantly increased the $\mathrm{Na}^{+}-\mathrm{K}^{+}$ATPase activity in both erythrocytes and sciatic nerve of STZ-induced diabetic rats, correlating with the improvement of sciatic NCV and thermal and mechanical hyperalgesia, which suggested that maltol may treat DPN by elevating the decreased $\mathrm{Na}^{+}-\mathrm{K}^{+}$-ATPase activity in diabetic rats.

\subsection{Effects of maltol on oxidative stress markers in STZ-induced diabetic rats}

In comparison with nondiabetic rats (Nor), STZ-induced diabetic rats (Con) showed a significant imbalance between oxidation and antioxidation with increased serum MDA levels and decreased TAOC, GSH, and SOD content in serum and sciatic nerves. In comparison with STZ-induced diabetic rats (Con), repeated administration of maltol for 12 weeks significantly increased the serum TAOC, GSH, and SOD levels and decreased the serum MDA level in a dose-dependent manner (Fig. 5A-D). Furthermore, maltol also increased the SOD activity $(p<0.01)$ and GSH content $(p<0.05)$ in sciatic nerves, especially at a dose of $200 \mathrm{mg} \mathrm{kg}^{-1}$ (Fig. 5E-F). Note that the antioxidative markers, TAOC, SOD, and GSH, both in serum and the sciatic nerves of maltol-treated (100 and $\left.200 \mathrm{mg} \mathrm{kg}^{-1}\right)$ rats were not different from those of nondiabetic rats (Nor), but the MDA level evidently was still higher than that of Nor, suggesting that an improvement in the imbalance between oxi- dation and antioxidation by maltol mainly were attributed to an increase in antioxidative ability.

Accumulating evidence has supported the finding that oxidative stress caused by chronic hyperglycemia was a key mechanism of DPN and seeking excellent antioxidative therapies was essential for the prevention of neuropathy in patients with diabetes. ${ }^{30}$ Oxidative stress occurred in a cellular system when the generation of free radicals exceeded their rate of utilization. ${ }^{31}$ It established a linkage between the physiological and metabolic initiators involved in the progressive nerve fiber injury, dysfunction, and loss during the development of DPN. ${ }^{32} \mathrm{GSH}$, the most sensitive index of ongoing oxidative stress, was a first line of defense against free radicals and the most potent endogenous antioxidant in most mammalian cells. Depletion of GSH significantly impaired the mitochondrial function of motor neurons and rendered them susceptible to oxidative damage and death, ${ }^{33}$ while sparing GSH by antioxidants was highly effective in protecting neuronal cells from damage. ${ }^{34}$ As a potent antioxidant, repeated treatment with maltol significantly increased the antioxidative capacity of diabetic rats by elevating the serum TAOC, SOD, and GSH levels and decreasing the serum MDA level, and increasing the SOD and GSH levels in the sciatic nerve, which suggested that maltol may treat DPN by protecting the sciatic nerve from oxidative damage in STZ-induced diabetic rats.

\subsection{Effects of maltol on caspase-3 activities in STZ-induced diabetic rats}

In comparison with nondiabetic rats (Nor), STZ-induced diabetic rats (Con) exhibited increased caspase-3 activity in DRG $(p<0.01)$, suggesting the occurrence of apoptosis. In comparison with STZ-induced diabetic rats (Con), repeated treatment with maltol for 12 weeks had no significant influence on the activity of caspase-3, but it separately produced a decrease by $14.6 \%, 15.4 \%$, and $23.3 \%$ at doses of 50,100 , and $200 \mathrm{mg} \mathrm{kg}^{-1}$. The caspase- 3 activities of rats in maltol-treated groups were higher than those of nondiabetic rats (Nor) (Fig. 6). These results suggested that treatment with maltol may inhibit cell apoptosis in DRG. 
A

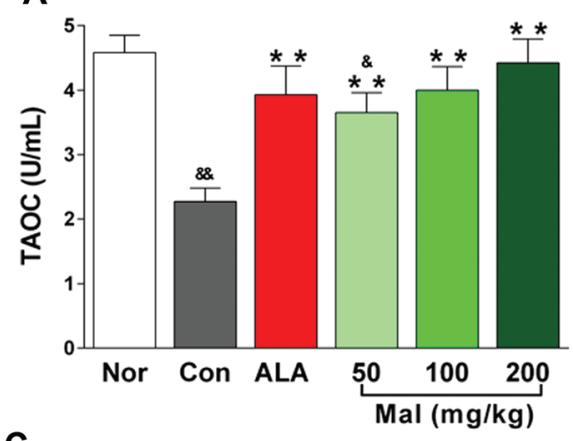

C

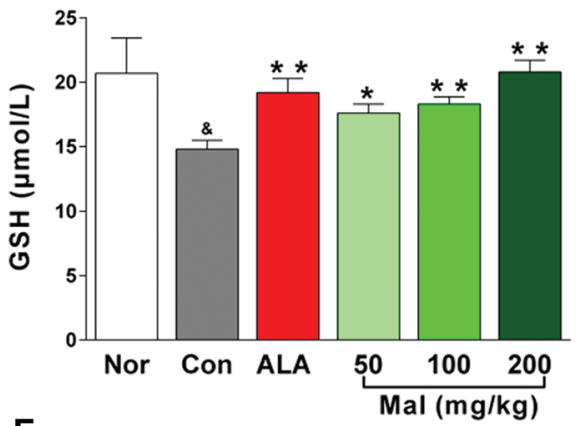

E

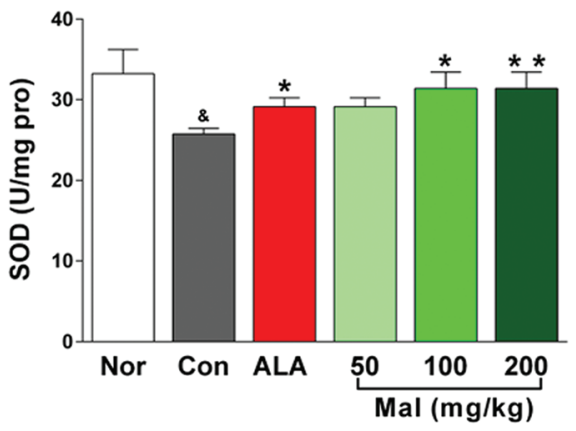

B

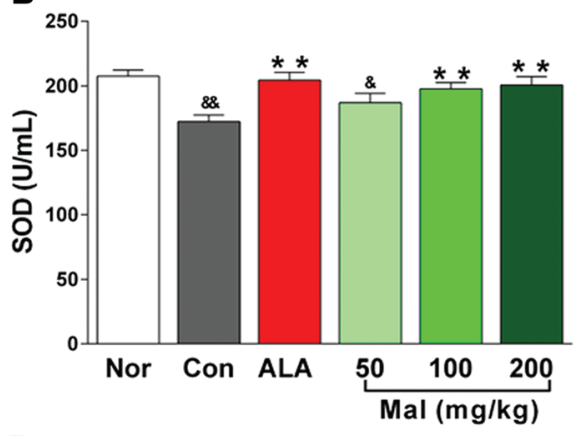

D

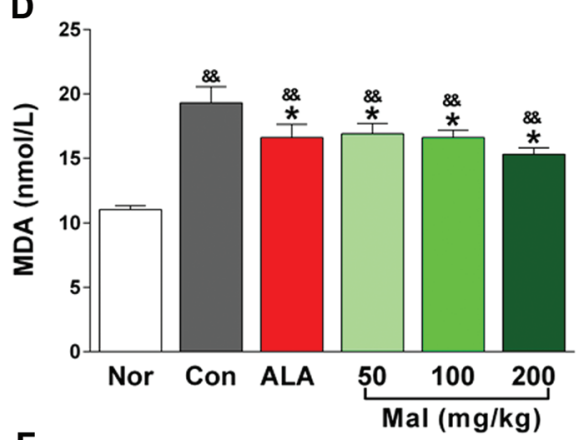

F

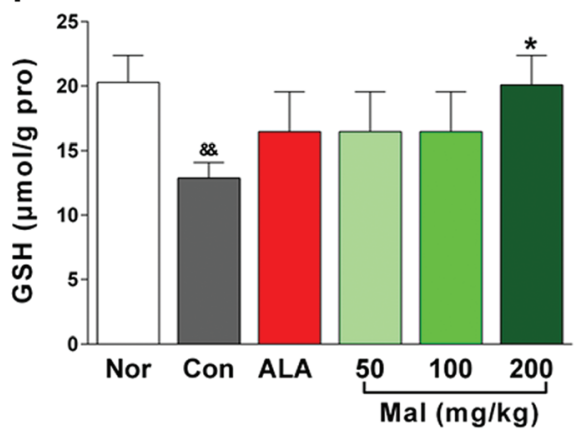

Fig. 5 Effects of treatment with maltol on the oxidative markers in STZ-induced diabetic rats. (A) TAOC in serum, (B) SOD in serum, (C) GSH in serum, (D) MDA in serum, (E) SOD in the sciatic nerve, and (F) GSH in the sciatic nerve. Data expressed as mean \pm SEM, $n=6-8$ for (A) and (C), $n=$ 9-11 for (B), $n=7-10$ for (D) and (F), $n=7-11$ for $(E),{ }^{\&} P<0.05,{ }^{\text {\&\& }} P<0.01$ vs. Nor; ${ }^{*} P<0.05,{ }^{* \star} P<0.01$ vs. Con.

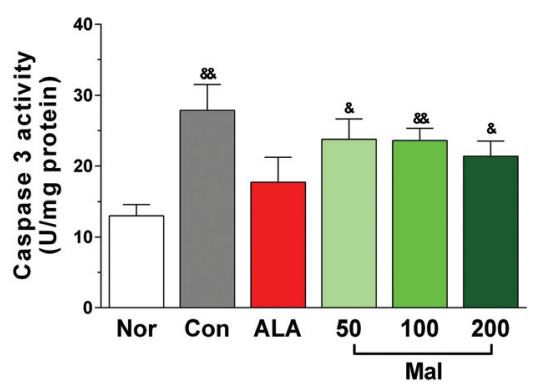

Fig. 6 Effect of treatment with maltol on the caspase-3 activity in DRG of STZ-induced diabetic rats. Data expressed as mean \pm SEM, $n=8-12$, \& $P<0.05$, \&\& $P<0.01$ vs. Nor.

The axons of DRG neurons were afferents that transmitted sensory information to the central nervous system. Due to the exuberant metabolism and super sensitivity to hyperglycemia, DRG neurons were more easily injured and subjected to apop- tosis during the progression of diabetes, ultimately leading to numbness and pain in patients. ${ }^{35}$ In addition, there were also close connections between SCs and DRG neurons that SCs played important roles in the neurite outgrowth of DRG neurons and a decrease of the nerve growth factor level secreted by SCs might cause a defect in axonal regeneration. ${ }^{36}$ Thus, DRG neurons were considered to be an important experimental object of DPN. Large amounts of research have reported that elevated glucose levels could produce oxidative damage to DRG neurons and push them to apoptosis, with the mitochondria serving as an important target. ${ }^{37,38}$ Caspase-3 belongs to the cysteine-aspartic acid protease family and is an important executor of cell apoptosis. To fully assess the effects of maltol treatment on apoptosis, we also collected the DRG neurons to determine the pro-apoptotic caspase-3 activities. We found that long-term treatment with maltol produced a decrease by at least $14.6 \%$, although without statistical significance, which was consistent with the expression of caspase-3 

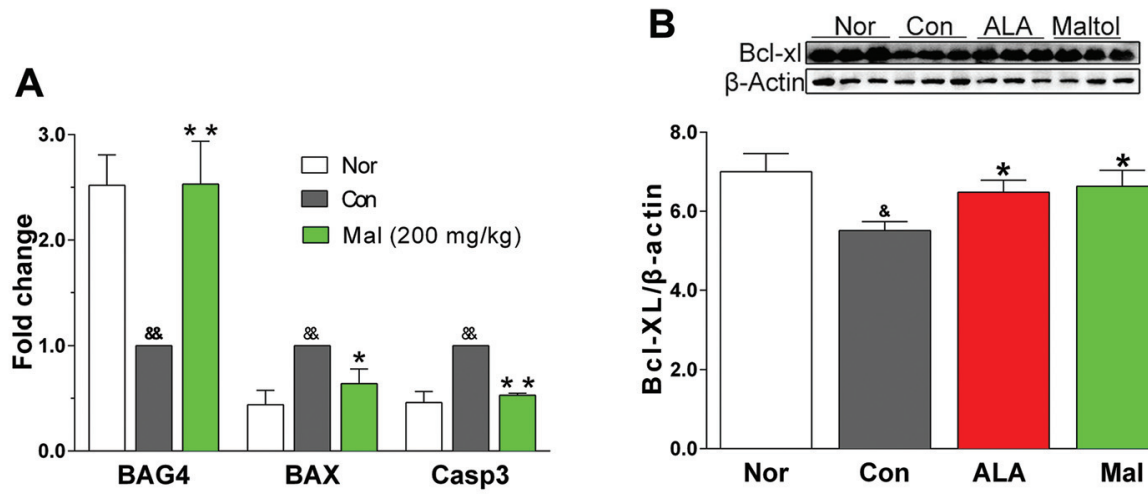

.
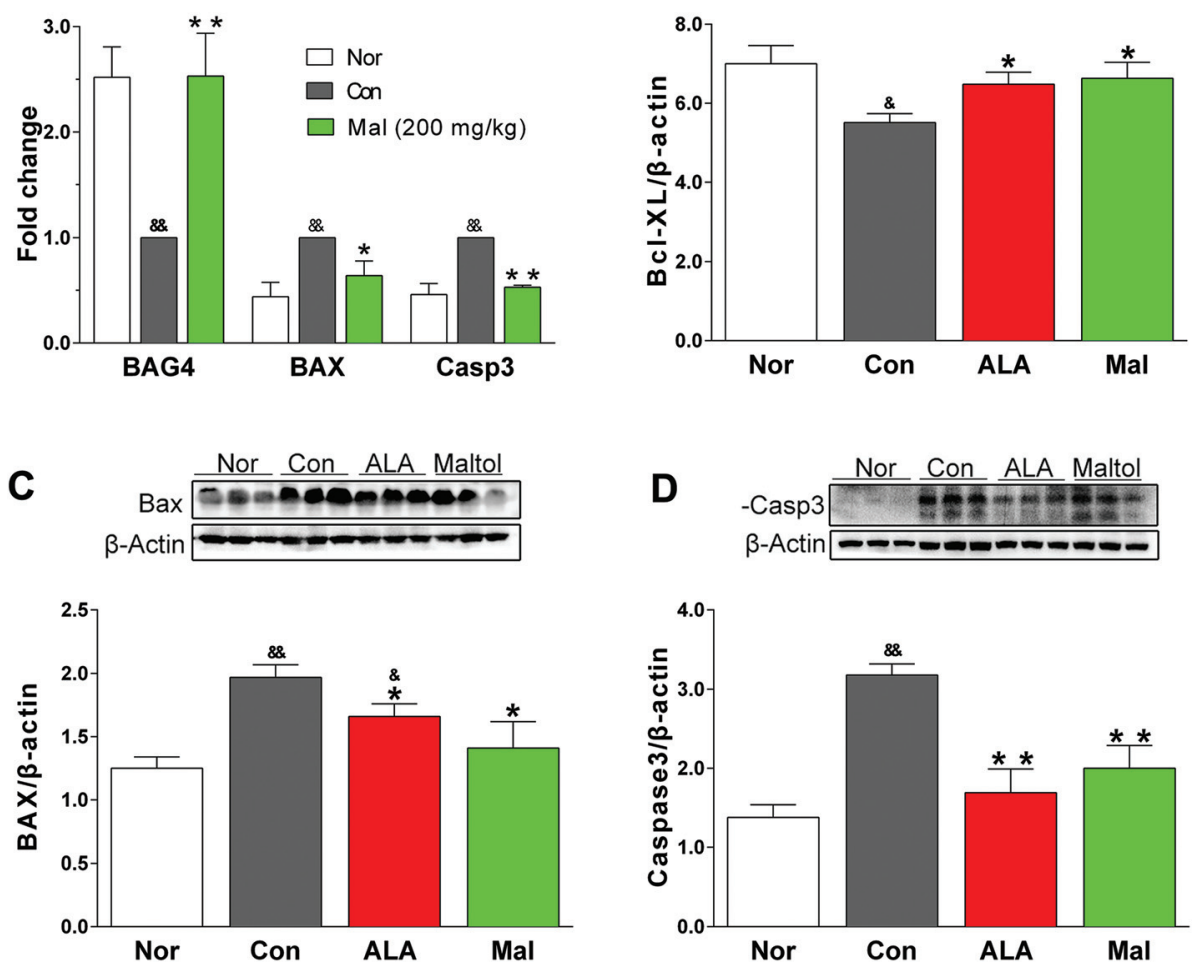

Fig. 7 Effects of maltol on the expression of genes and proteins related to apoptosis in sciatic nerves of STZ-induced diabetic rats. (A) The expression of BAG4, BAX, and caspase-3 genes, (B) the expression of $\mathrm{Bcl}-\mathrm{XL}$ protein, (C) the expression of BAX protein, and (D) the expression of caspase-3 protein. Data expressed as mean \pm SEM, $n=5-6$ for (A), and $n=3$ for (B), (C), and (D), ${ }^{\&} P<0.05,{ }^{\text {\& } ~} P<0.01$ vs. Nor; $P<0.05,{ }^{* *} P<$ 0.01 vs. Con.

in sciatic nerves shown in Fig. 7, suggesting that maltol may inhibit cell apoptosis to improve the peripheral nerve function.

\subsection{Effects of maltol on the expression of apoptosis-related proteins in STZ-induced diabetic rats}

BAG4 and Bcl-XL were two major negative regulators of apoptotic pathways, whereas BAX and caspase-3 were important proapoptotic proteins. In comparison with nondiabetic rats (Nor), STZ-induced diabetic rats (Con) displayed increased expressions of BAX and caspase-3 and decreased expressions of BAG4 and Bcl-XL, suggesting activation of the apoptotic pathway. In comparison with STZ-induced diabetic rats (Con), repeated treatment with maltol for 12 weeks significantly lowered the expression of BAX and caspase- 3 both in gene and protein levels, and it remarkably elevated the expression of BAG4 gene and Bcl-XL protein. Furthermore, the expressions of BAX, caspase-3, BAG4, and Bcl-XL in the maltol-treated group were nearly equivalent to the expressions of nondiabetic rats (Nor) (Fig. 7).

Oxidative stress was closely associated with apoptosis in many cells by increasing the peroxidation of membrane lipids, impairing the functional proteins and signal transduction pathways, and changing the cellular redox potential. ${ }^{39}$ The pathogenesis of DPN involved demyelination, axonal atrophy, and degeneration, indicating that apoptosis participated in its occurrence and development. ${ }^{39}$ The sciatic nerve is the longest and largest peripheral nerve in the body, and we analyzed the expressions of apoptosis-associated proteins in the sciatic nerves of diabetic rats to further investigate how maltol treated DPN. Bcl-XL and BAX were two important proteins in cell survival, whereas Bcl-XL belonged to the pro-survival Bcl-2 family and could bind to the pro-apoptotic protein BAX to suppress the induction of apoptosis. ${ }^{40}$ Our study showed that repeated administration of maltol significantly decreased the expression of BAX and increased the expression of Bcl-XL of sciatic nerves in STZ-induced diabetic rats, and evidently decreased the expression of caspase-3. The results suggested that maltol may improve the nerve function by inhibiting cell apoptosis.

\subsection{Effects of maltol on the expression of apoptosis-related proteins in RSC96 cells injured by $\mathrm{H}_{2} \mathrm{O}_{2}$}

Schwann cells (SCs) accounted for most of the peripheral nervous system and RSC96 cells belonged to the rat Schwann cell line. We used RSC96 cells to evaluate the effect of maltol in the present study. $\mathrm{H}_{2} \mathrm{O}_{2}$ is a commonly used oxidant to induce cell apoptosis through the activation of oxidative stress. In comparison with the cells without $\mathrm{H}_{2} \mathrm{O}_{2}$ treatment (Nor), 
A

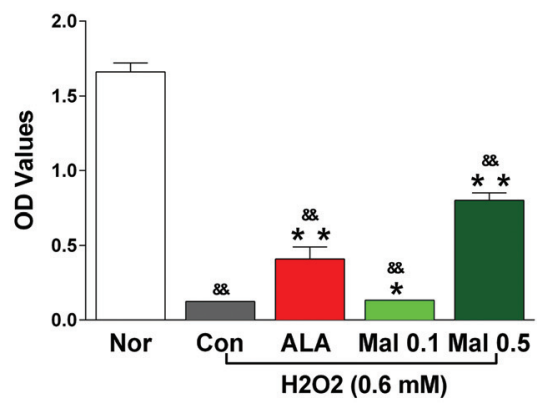

B

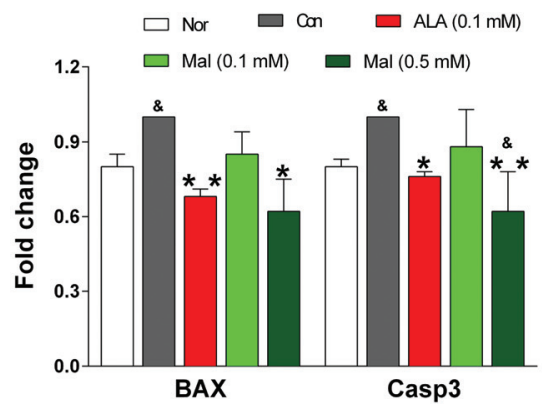

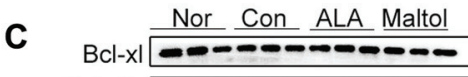

B-Actin

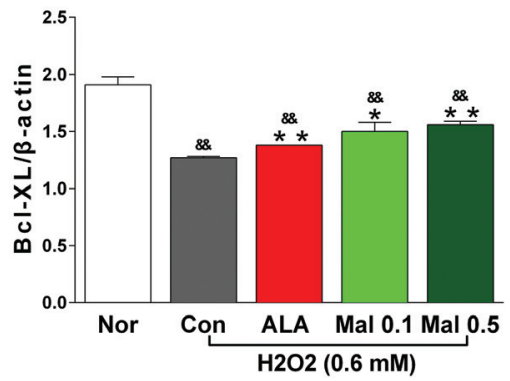

Fig. 8 Effects of maltol on the viability of RSC96 cells injured by $\mathrm{H}_{2} \mathrm{O}_{2}$ and the expression of genes and proteins related to apoptosis. (A) Cell viability, (B) the expression of BAX and caspase-3 gene, (C) the expression of Bcl-XL protein. Data expressed as mean \pm SEM, $n=5$ for (A), $n=3-6$ for (B) and (C), ${ }^{\&} P<0.05,{ }^{\text {\&\& }} P<0.01$ vs. Nor; ${ }^{*} P<0.05$, ${ }^{* *} P<0.01$ vs. Con.

$\mathrm{H}_{2} \mathrm{O}_{2}$-treated (0.6 mM) cells (Con) showed a significant decrease in viability and anti-apoptotic protein Bcl-XL expression as well as an evident increase in pro-apoptotic protein BAX and caspase-3 expression, suggesting the apoptosis of RSC96 cells. In comparison with the $\mathrm{H}_{2} \mathrm{O}_{2}$-treated cells (Con), maltol at concentrations of 0.1 and $0.5 \mathrm{mM}$ significantly increased the viability of RSC96 cells after coincubation with $\mathrm{H}_{2} \mathrm{O}_{2}$ (Fig. 8A), evidently elevated the expression of Bcl-XL protein in a dose-dependent manner (Fig. 8B), and decreased the expression of BAX and caspase-3 genes (Fig. 8C), although the cell viability and expression of Bcl-XL remained lower than that of the cells without $\mathrm{H}_{2} \mathrm{O}_{2}$ treatment (Nor). The results suggested that maltol may inhibit the $\mathrm{H}_{2} \mathrm{O}_{2}$-induced oxidative injury to RSC96 cells.

SCs, the only glial cells in the peripheral nervous system, generated myelin and supported the structural and functional integrity of nerves. ${ }^{41}$ The disruption to SCs' metabolism triggered by hyperglycemia resulted in an accumulation of neurotoxic intermediates, which contributed to axonal degeneration, endothelial dysfunction, and diabetic neuropathy. ${ }^{42}$ Furthermore, SCs also imposed cellular specializations that allowed for the fast conduction of action potentials. ${ }^{43}$ Reduced NVC in DPN was largely due to the impaired function of SCs, which generated and maintained a multilamellar insulating myelin sheath around an associated axon and provided a permissive environment for peripheral nerve regeneration. ${ }^{44}$ Previous studies showed that the exposure of SCs to $\mathrm{H}_{2} \mathrm{O}_{2}$ in vitro significantly caused oxidative stress and induced apoptosis via the mitochondrial pathway. ${ }^{45}$ In the present study, we incubated RSC96 cells with maltol and $\mathrm{H}_{2} \mathrm{O}_{2}(0.6 \mathrm{mM})$ to investigate the effect of maltol on the apoptosis induced by oxidative stress. A previous study revealed that maltol could help SCs recover from $\mathrm{H}_{2} \mathrm{O}_{2}$-induced oxidative injury and protect SCs from apoptosis in vitro, suggesting it may be a potential neuroprotective therapeutic agent for oxidativestress-induced injury. ${ }^{46}$ Our study found that maltol significantly increased the viability of RSC96 cells after incubation with $\mathrm{H}_{2} \mathrm{O}_{2}$, remarkably decreased the expressions of pro-apop- totic BAX and caspase-3, and elevated the expression of antiapoptotic Bcl-XL in a dose-dependent manner.

Studies have reported, however, that maltol (1.2 mM) induced an apoptotic cell death together with iron(II) sulfate $\left(\mathrm{FeSO}_{4} ; 0.4 \mathrm{mM}\right)$ in HL60 cells, which may have resulted from the generation of reactive oxygen species by redox cycling, but maltol or $\mathrm{FeSO}_{4}$ alone did not affect the cells. ${ }^{47}$ Hironishi et al. found that maltol induced apoptosis of neuroblastoma cell lines and primary murine fetal hippocampal neurons early in $1996,{ }^{48}$ and recent studies showed that the cytotoxicity of maltol was not the result of the apoptotic pathway in some human tumor cell lines. ${ }^{49}$ In our study, we found that maltol at concentrations of 0.1 and $0.5 \mathrm{mM}$ protected RSC96 cells from $\mathrm{H}_{2} \mathrm{O}_{2}$-induced apoptosis. Thus, we thought that the influence of maltol on cells, (i.e., benefit or toxicity) may lay in its concentration in the cells and the cell types.

\section{Conclusion}

As a common flavor-enhancing agent and metal ion chelator, maltol also possesses a strong antioxidative activity. The present study first found that repeated treatment with maltol at doses ranging from $50 \mathrm{mg} \mathrm{kg}^{-1}$ to $200 \mathrm{mg} \mathrm{kg}^{-1}$, selected according to previous research, ${ }^{7,8,50}$ in STZ-induced diabetic rats produced the following results: (1) significantly enhanced the conduction velocity of the sciatic nerve, (2) improved the thermal and mechanical hyperalgesia, (3) increased $\mathrm{Na}^{+}-\mathrm{K}^{+}$ATPase activity, (4) elevated the antioxidative ability, (5) inhibited the apoptosis of the sciatic nerve in diabetic rats and apoptosis induced by $\mathrm{H}_{2} \mathrm{O}_{2}$ in RSC96 cells, and (6) decreased the caspase- 3 activities in DRG, but (7) had no significant influences on the FBG level and BW. These results suggested that the benefits of maltol on DPN were not attributed to the control of blood glucose but rather to improvements in nerve function through antioxidative stress and apoptosis.

We found maltol to be effective in slowing the progression of DPN without affecting hyperglycemia in STZ-induced dia- 
betic rats. These benefits may be associated with an increase in $\mathrm{Na}^{+}-\mathrm{K}^{+}$-ATPase activity, amelioration of oxidative stress, and inhibition of apoptosis. Thus, maltol may provide a new alternative for the treatment of DPN.

\section{Author contributions}

N. G. and C. L. designed the entire study, performed most of the experiments and prepared the manuscript. Q. L. and S. L. participated in the measurement of thermal and mechanical thresholds. Y. H. and X. W. took part in monitoring the MNCV. G. B. and M. Y. determined the oxidative stress-related index. S. S. was in charge of the dosages in the animal experiment. C. X. supplied the maltol. Z. S. supplied academic and technical support. Both Z. S. and C. L. are the corresponding authors.

\section{Conflicts of interest}

There are no conflicts of interest to declare.

\section{Acknowledgements}

This work was supported by the CAMS Initiative for Innovative Medicine (CAMS-I2 M) under No. 2016-I2M-2-006 and No. 2017-I2M-1-010. We thank LetPub for its linguistic assistance during the preparation of this manuscript.

\section{References}

1 M. Brownlee, Biochemistry and molecular cell biology of diabetic complications, Nature, 2001, 414, 813-820.

2 E. Agathos, A. Tentolouris, I. Eleftheriadou, P. Katsaouni, I. Nemtzas, A. Petrou, C. Papanikolaou and N. Tentolouris, Effect of $\alpha$-lipoic acid on symptoms and quality of life in patients with painful diabetic neuropathy. Journal of international medical research, J. Int. Med. Res., 2018, 46, 17791790.

3 S. G. Sayyed, A. Kumar and S. S. Sharma, Effects of U83836E on nerve functions, hyperalgesia and oxidative stress in experimental diabetic neuropathy, Life Sci., 2006, 79, 777-783.

4 H. C. Jeong, H. D. Hong, Y. C. Kim, Y. K. Rhee, S. Y. Choi, K. T. Kim, S. S. Kim, Y. C. Lee and C. W. Cho, Quantification of maltol in Korean ginseng (Panax ginseng) products by high-performance liquid chromatographydiode array detector, Pharmacogn. Mag., 2015, 11, 657-664.

5 K. G. Lee and T. Shibamoto, Antioxidant properties of aroma compounds isolated from soybeans and mung beans, J. Agric. Food Chem., 2000, 48, 4290-4293.

6 K. S. Kang, H. Y. Kim, J. S. Pyo and T. Yokozawa, Increase in the free radical scavenging activity of ginseng by heatprocessing, Biol. Pharm. Bull., 2006, 29, 750-754.
7 K. S. Kang, N. Yamabe, H. Y. Kim and T. Yokozawa, Role of maltol in advanced glycation end products and free radicals: in vitro and in vivo studies, J. Pharm. Pharmacol., 2008, 60, 445-452.

8 Y. Han, Q. Xu, J. N. Hu, X. Y. Han, W. Li and L. C. Zhao, Maltol, a food flavoring agent, attenuates acute alcoholinduced oxidative damage in mice, Nutrients, 2015, 7, 682-696.

9 Y. Song, S. Hong, Y. Iizuka, C. Y. Kim and G. J. Seong, The neuroprotective effect of maltol against oxidative stress on rat retinal neuronal cells, Korean J. Ophthalmol., 2015, 29, 58-65.

10 Y. Yang, J. Wang, C. Xu, H. Pan and Z. Zhang, Maltol inhibits apoptosis of human neuroblastoma cells induced by hydrogen peroxide, J. Biochem. Mol. Biol., 2006, 39, 145149.

11 X. Yu, L. Zhang, X. Yang, H. Huang, Z. Huang, L. Shi, H. Zhang and G. Du, Salvianolic acid A protects the peripheral nerve function in diabetic rats through regulation of the AMPK-PGC1 $\alpha$-Sirt3 axis, Molecules, 2012, 17, 1121611228.

12 H. N. Kim, Y. R. Kim, J. Y. Jang, H. K. Shin and B. T. Choi, Electroacupuncture inhibits phosphorylation of spinal phosphatidylinositol 3-kinase/Akt in a carrageenaninduced inflammatory rat model, Brain Res. Bull., 2012, 87, 199-204.

13 S. Kumar, K. H. Arun, C. L. Kaul and S. S. Sharma, Effects of adenosine and adenosine A2A receptor agonist on motor nerve conduction velocity and nerve blood flow in experimental diabetic neuropathy, Neurol. Res., 2005, 27, 60-66.

14 R. Stavniichuk, V. R. Drel, H. Shevalye, Y. Maksimchyk, T. M. Kuchmerovska, J. L. Nadler and I. G. Obrosova, Baicalein alleviates diabetic peripheral neuropathy through inhibition of oxidative-nitrosative stress and p38 MAPK activation, Exp. Neurol., 2011, 230, 106-113.

15 T. Brismar, A. A. Sima and D. A. Greene, Reversible and irreversible nodal dysfunction in diabetic neuropathy, Ann. Neurol., 1987, 21, 504-507.

16 C. G. Jolivalt, K. E. Frizzi, L. Guernsey, A. Marquez, J. Ochoa, M. Rodriguez and N. A. Calcutt, Peripheral Neuropathy in Mouse Models of Diabetes, Curr. Protoc. Mouse Biol., 2016, 6, 223-255.

17 N. Papanas and D. Ziegler, Efficacy of alpha-lipoic acid in diabetic neuropathy, Expert Opin. Pharmacother., 2014, 15, 2721-2731.

18 I. G. Obrosova, Diabetic painful and insensate neuropathy: pathogenesis and potential treatments, Neurotherapeutics, 2009, 6, 638-647.

19 S. O. Kim and H. J. Kim, Berberine ameliorates cold and mechanical allodynia in a rat model of diabetic neuropathy, J. Med. Food, 2013, 16, 511-517.

20 J. Han, P. Tan, Z. Li, Y. Wu, C. Li, Y. Wang, B. Wang, S. Zhao and Y. Liu, Fuzi attenuates diabetic neuropathy in rats and protects Schwann cells from apoptosis induced by high glucose, PLoS One, 2014, 9, e86539.

21 X. Yang, F. Liu, J. Guo, W. Yao, Q. Li, T. Liu and L. Xu, Antioxidation and anti-inflammatory activity of Tang $\mathrm{Bi}$ 
Kang in rats with diabetic peripheral neuropathy, $B M C$ Complementary Altern. Med., 2015, 15, 66-75.

22 E. P. Davidson, L. J. Coppey, B. Dake and M. A. Yorek, Treatment of streptozotocin-induced diabetic rats with alogliptin: effect on vascular and neural complications, Exp. Diabetes Res., 2011, 2011, 810469.

23 E. P. Davidson, L. J. Coppey, A. Holmes and M. A. Yorek, Effect of inhibition of angiotensin converting enzyme and/ or neutral endopeptidase on vascular and neural complications in high fat fed/low dose streptozotocin-diabetic rats, Eur. J. Pharmacol., 2012, 677, 180-187.

24 N. A. Calcutt, Modeling diabetic sensory neuropathy in rats, Methods Mol. Med., 2004, 99, 55-65.

25 N. A. Calcutt, M. C. Jorge, T. L. Yaksh and S. R. Chaplan, Tactile allodynia and formalin hyperalgesia in streptozotocin-diabetic rats: effects of insulin, aldose reductase inhibition and lidocaine, Pain, 1996, 68, 293-299.

26 S. Kambiz, J. W. van Neck, S. G. Cosgun, M. H. van Velzen, J. A. Janssen, N. Avazverdi, S. E. Hovius and E. T. Walbeehm, An early diagnostic tool for diabetic peripheral neuropathy in rats, PLoS One, 2015, 10, e0126892.

27 D. Raccah, M. F. Lamotte-Jannot, T. Issautier and P. Vague, Effect of experimental diabetes on Na/K-ATPase activity in red blood cells, peripheral nerve and kidney, Diabete Metab., 1994, 20, 271-274.

28 P. Vague, T. C. Coste, M. F. Jannot, D. Raccah and M. Tsimaratos, C-peptide, $\mathrm{Na}^{+}, \mathrm{K}^{+}$-ATPase, and diabetes, Exp. Diabesity Res., 2004, 5, 37-50.

29 D. Raccah, P. Gallice, J. Pouget and P. Vague, Hypothesis: low $\mathrm{Na} / \mathrm{K}$-ATPase activity in the red cell membrane, a potential marker of the predisposition to diabetic neuropathy, Diabete Metab., 1992, 18, 236-241.

30 A. M. Schmeichel, J. D. Schmelzer and P. A. Low, Oxidative injury and apoptosis of dorsal root ganglion neurons in chronic experimental diabetic neuropathy, Diabetes, 2003, 52, 165-171.

31 A. M. Vincent, J. W. Russell, P. Low and E. L. Feldman, Oxidative stress in the pathogenesis of diabetic neuropathy, Endocr. Rev., 2004, 25, 612-628.

32 A. Hosseini and M. Abdollahi, Diabetic Neuropathy and Oxidative Stress: Therapeutic Perspectives, Oxid. Med. Cell. Longevity, 2013, 2013, 168039.

33 M. Rizzardini, M. Lupi, S. Bernasconi, A. Mangolini and L. Cantoni, Mitochondrial dysfunction and death in motor neurons exposed to the glutathione-depleting agent ethacrynic acid, J. Neurol. Sci., 2003, 207, 51-58.

34 M. S. Kobayashi, D. Han and L. Packer, Antioxidants and herbal extracts protect HT-4 neuronal cells against glutamate-induced cytotoxicity, Free Radical Res., 2000, 32, 115-124.

35 J. M. McHugh and W. B. McHugh, Diabetes and peripheral sensory neurons: what we don't know and how it can hurt us, AACN Clin. Issues, 2004, 15, 136-149.

36 T. Tosaki, H. Kamiya, Y. Yasuda, K. Naruse, K. Kato, M. Kozakae, N. Nakamura, T. Shibata, Y. Hamada,
E. Nakashima, Y. Oiso and J. Nakamura, Reduced NGF secretion by Schwann cells under the high glucose condition decreases neurite outgrowth of DRG neurons, Exp. Neurol., 2008, 213, 381-387.

37 A. M. Vincent, L. L. McLean, C. Backus and E. L. Feldman, Short-term hyperglycemia produces oxidative damage and apoptosis in neurons, FASEB J., 2005, 19, 638-640.

38 A. M. Schmeichel, J. D. Schmelzer and P. A. Low, Oxidative injury and apoptosis of dorsal root ganglion neurons in chronic experimental diabetic neuropathy, Diabetes, 2003, 52, 165-171.

39 S. S. Kamboj, R. K. Vasishta and R. Sandhir, N-acetylcysteine inhibits hyperglycemia-induced oxidative stress and apoptosis markers in diabetic neuropathy, J. Neurochem., 2010, 112, 77-91.

40 S. Murai, J. Matuszkiewicz, Y. Okuzono, H. Miya and R. De Jong, Aurora B Inhibitor TAK-901 Synergizes with BCL-xL Inhibition by Inducing Active BAX in Cancer Cells, Anticancer Res., 2017, 37, 437-444.

41 K. R. Monk, M. L. Feltri and C. Taveggia, New insights on Schwann cell development, Glia, 2015, 63, 1376-1393.

42 N. P. Gonçalves, C. B. Vægter, H. Andersen, L. Østergaard, N. A. Calcutt and T. S. Jensen, Schwann cell interactions with axons and microvessels in diabetic neuropathy, Nat. Rev. Neurol., 2017, 13, 135-147.

43 Z. L. Chen, W. M. Yu and S. Strickland, Peripheral regeneration, Annu. Rev. Neurosci., 2007, 30, 209-233.

44 L. Eckersley, Role of the Schwann cell in diabetic neuropathy. International review of neurobiology, Int. Rev. Neurobiol., 2002, 50, 293-321.

45 J. Ma, J. Liu, Q. Wang, H. Yu, Y. Chen and L. Xiang, The beneficial effect of ginsenoside Rg1 on Schwann cells subjected to hydrogen peroxide induced oxidative injury, Int. J. Biol. Sci., 2013, 9, 624-636.

46 S. Hong, Y. Iizuka, T. Lee, C. Y. Kim and G. J. Seong, Neuroprotective and neurite outgrowth effects of maltol on retinal ganglion cells under oxidative stress, Mol. Vision, 2014, 20, 1456-1462.

47 K. Murakami, K. Ishida, K. Watakabe, R. Tsubouchi, M. Naruse and M. Yoshino, Maltol/iron-mediated apoptosis in HL60 cells: participation of reactive oxygen species, Toxicol. Lett., 2006, 161, 102-107.

48 M. Hironishi, R. Kordek, R. Yanaqihara and R. M. Garruto, Maltol (3-hydroxy-2-methyl-4-pyrone) toxicity in neuroblastoma cell lines and primary murine fetal hippocampal neuronal cultures, Neurodegeneration, 1996, 5, 325-329.

49 E. Yasumoto, K. Nakano, T. Nakayachi, S. R. Morshed, K. Hashimoto, H. Kikuchi, H. Nishikawa, M. Kawase and H. Sakagami, Cytotoxic activity of deferiprone, maltol and related hydroxyketones against human tumor cell lines, Anticancer Res., 2004, 24, 755-762.

50 Y. B. Kim, S. H. Oh, D. E. Sok and M. R. Kim, Neuroprotective effect of maltol against oxidative stress in brain of mice challenged with kainic acid, Nutr. Neurosci., 2004, 7, 33-39. 